\title{
News on the Calibration of Non-Automatic Weighing Instruments
}

\author{
Nieves Medina ${ }^{1, *}$ \\ ${ }^{1}$ Centro Español de Metrología, Tres Cantos, 28760, Madrid, Spain
}

\begin{abstract}
EURAMET Calibration Guide No. 18 'Guidelines on the Calibration of Non-Automatic Weighing Instruments' is the document used, not only in Europe but worldwide, as a reference for the calibration of non-automatic weighing instruments. The purpose of the guide is to enhance the equivalence and mutual recognition of calibration results obtained by laboratories performing calibrations of non-automatic weighing instruments. It contains advice on general aspects of calibration, measurement methods and results, including uncertainty of measurement, the calibration certificate and the further use of the instrument. EURAMET's Calibration Guide No. 18 latest update has been the first one involving direct collaboration with balance manufacturers. In this new version the guide has been improved in many technical details with the aim of clarifying the content of the guide, including some necessary details or simplifying some calculations.
\end{abstract}

\section{Introduction}

The purpose of this guide [1] is to improve the equivalence and mutual recognition of calibration results obtained by laboratories that perform calibrations of non-automatic weighing instruments. Its content includes detailed information on general aspects of calibration, measurement methods and measurement results, including their associated uncertainty, the calibration certificate and the use of conventional mass and mass values.

This guide emerged as a calibration guide within the EA European Accreditation Body. The reason is that there were discrepancies in the comparisons, so it was necessary to obtain a calibration method that was sufficient to adequately characterize the instrument and obtain compatible results among the laboratories.

In 2009, it became dependent on EURAMET as well as all EA calibration guides, which also became dependent on EURAMET.

This guide has undergone three revisions, in 2010, in 2011 (this only due to an internal issue of EURAMET as an organization) and the most recent in 2015.

In 2011, a EURAMET project began to review the contents of the guide. A group of manufacturers and calibration laboratories that found deficiencies in it requested this review and, consequently, a working group with representatives of the National Metrology

\footnotetext{
* Corresponding author: mnmedina@cem.es
} 
Institutes and Accreditation Bodies and manufacturers of weighing instruments was created to carry out the review.

The guide has become one of the most required documents of EURAMET, it has even been adopted by other Regional Metrological Organizations and is widely used as a base document with presumption of conformity by the accreditation bodies worldwide.

\section{Description}

Through the revision, the improvements discussed below have been achieved. In the first place, necessary aspects, which had not been contemplated before, have been addressed. Thus, specific considerations have been included for the multi-range instruments, the determination of the minimum weight and certain aspects on the adjustment and correction by air buoyancy have been specified.

On the other hand, confusing treatments such as the use of substitution loads and the different valid forms that exist to perform the eccentricity test and the determination of the relationship between the errors and the indications have been clarified.

Finally, an attempt has also been made to facilitate its application, thus new considerations regarding air density have been included, especially in the determination of its uncertainty, and the examples included are now more complete, including the two possible cases depending on whether or not the adjustment is performed before calibration. A new example has been included to illustrate the methodology for the matrix determination of the approximation function between errors and indications.

We will next explain what these improvements consist of.

\subsection{Considerations for multiple range instruments}

In the new version of the guide, the following considerations are specifically included for the multiple range instruments, which were not included in the previous version:

- Independent calibration results are obtained for each range.

- The eccentricity test is only performed for the highest range.

- In the repeatability test, it is sufficient to use a load that is lower and closer to the maximum range that corresponds to the smallest step.

\subsection{Determination of minimum weight}

In general, mass standards below $1 \mathrm{mg}$ are not available, OIML $\mathrm{R} 111$ [2] does not contemplate them. There are non-automatic weighing instruments that have ranges well below milligram. These instruments are called microbalances and, in some cases, they can even have resolutions of the tenth of a microgram. These instruments are common, for example, in the pharmaceutical industry and the have a linear behaviour.

It is a general practice for users to establish specific requirements on the behaviour of an instrument (user requirements specifications). They usually define upper limits for the measurement uncertainty values that are acceptable for a given weighing application. Frequently users also have to comply with regulations that stipulate the fulfilment of a certain requirement on the uncertainty of measurement. Normally, these requirements are indicated as relative values, for example compliance with a measurement uncertainty of $0.1 \%$.

For weighing instruments, global uncertainty is usually used to determine whether the instrument meets certain user requirements. The global uncertainty is usually approximated by the linear equation, 


$$
U_{\mathrm{gl}}(W)=\alpha_{\mathrm{gl}}+\beta_{\mathrm{gl}} \cdot R
$$

where $R$ is the indication, $W$ the weighing result and $\alpha_{\mathrm{gl}}$ and $\beta_{\mathrm{gl}}$ are parameters to be determined. For a certain requirement of accuracy, Req, only the weightings with

$$
U_{\mathrm{gl}}(W) / R \leq \operatorname{Req}
$$

meet the respective user requirements. Therefore, only weightings with such a reading that

$$
R \geq \frac{\alpha_{\mathrm{gl}}}{\operatorname{Req}-\beta_{\mathrm{gl}}}
$$

have a relative measurement uncertainty lower than the specific requirement established by the user and are, therefore, acceptable. The limit value, that is, the lowest weighing result that satisfies the user requirement is

$$
R_{\min }=\frac{\alpha_{\mathrm{gl}}}{R e q-\beta_{\mathrm{gl}}}
$$

and it is called minimum weight. Based on this value, the user is able to define adequate standardized work procedures that ensure that the weightings made using the instrument meet the minimum weight requirement, that is, that only weighs quantities that have a mass greater than the minimum weight. The minimum weight is defined as the minimum amount of sample necessary for a weighing to achieve a specified accuracy of a specified weighing [3].

As the uncertainty of measurement in use may be difficult to estimate due to environmental factors such as high vibration levels, air currents, influences induced, or due to specific influences of the weighing application, such as samples electrostatically charged, magnetic stirrers, etc., a safety factor is usually applied.

The security factor $S F$ is a number greater than one, by which the user requirement $R e q$ is divided. The objective is to ensure that the global relative measurement uncertainty is less than or equal to the Req user requirement, divided by the safety factor. This ensures that the environmental effects, or the effects due to a particular application of weighing, that have an important effect on the measurement and that could, therefore, increase the uncertainty of weighing measurement beyond the level estimated by the global uncertainty, still allow (with a high degree of security) that the user requirement $R e q$ is fulfilled.

$$
U_{\mathrm{gl}}(W) / R<\operatorname{Req} / S F
$$

Therefore, the minimum weight based on the safety factor can be calculated as

$$
R_{\mathrm{min}, \mathrm{SF}}=\frac{\alpha_{\mathrm{gl}} \cdot S F}{R e q-\beta_{\mathrm{gl}} \cdot S F}
$$

The user has the responsibility to define the safety factor depending on the degree to which environmental effects and the application of specific weighing can influence the uncertainty of measurement. It must be taken into account that the minimum weight refers to the net weight (sample weight) that is weighed in the instrument, that is, the mass of the tare container must not be taken into account to meet the user requirement Req. Therefore, the minimum weight is often referred to as "minimum sample weight". 


\subsection{Performing the eccentricity test}

In the new version of the guide, the different ways of performing the eccentricity test have been specified. Four possible cases have been included:

- Case 1. Before the test the indication is set to zero. The test load is placed in the central position and then placed in the other four positions of the ends in arbitrary order.

- Case 2. The test load is placed in the central position and the instrument is tared. Then the test load moves in the other four positions of the ends in arbitrary order.

- Case 3. Before the test the indication is set to zero. The test load is placed in the centre position, removed, then placed in the next position, removed, and so on.

- Case 4. The test load is placed in the central position and the instrument is tared. Then the test load is placed in the next position, it is returned to the central position. Then it is placed in another position and put back in the centre position and so on.

The guide establishes the ways to determine the eccentricity in each case.

\subsection{Use of substitution loads}

Substitution loads are used if it is not possible to use mass standards to calibrate the entire range of the instrument. This is a very normal case in powerful instruments such as bridge scales.

The procedure consists of using substitution loads whose value is determined using the instrument in calibration as a comparator to adjust the substitution load so that it originates approximately the same indication as with mass standards.

The new version of the guide includes the case in which additional test loads are used, once the substitution has been made, using additionally the different mass standards used to determine the substitution loads or groups thereof separately. Consequently, the determination of uncertainty has also been adapted, where the correlation between mass standards has been taken into account.

\subsection{Characteristic of the weighing range}

Other changes made in the new version affect the determination of the approximation function that establishes the relationship between errors and indications. Annex C describes how to perform the approach based on the minimum chi-square approach. This approach is expressed by the following condition:

$$
\sum v_{j}^{2}=\sum\left(f\left(I_{j}\right)-E_{j}\right)^{2}=\text { minimum }
$$

where for each calibration point $j, p_{j}$ is the weighting factor (basically proportional to the inverse of the variances for each point), $E_{j}$ are the errors, the $I_{j}$ are the indications and $f$ is the corresponding approximation function.

In both versions the approximation is considered valid if it is satisfied that the value of the chi-square using the approximation function to be evaluated fulfils the following condition,

$$
\chi^{2} \text { obs } \leq v
$$

where $v=n-n_{\text {par }}$ being $n$ the number of calibration points and $n_{\text {par }}$ is the number of parameters of the approach function.

In the new version, another condition of consistency of the data with the proposed model appears, which is more restrictive, and it is given by this expression, 


$$
\max \left(\frac{\left|f\left(I_{j}\right)-E_{j}\right|}{U\left(f\left(I_{j}\right)\right)}\right)<1
$$

That is, the maximum value of all these quotients for each point $j$ with the previous notations and where $U$ is the expanded uncertainty, must be less than 1 .

In the case that the approximation is a polynomial, the minimum chi-square approach is transformed into a matrix equation.

In the new version, the expression for the weighting matrix appears for the first time, which is the inverse of a matrix that is the sum of three matrices:

- The first matrix considers the contributions to the uncertainty of the load, including the contributions for mass calibration, corresponding drift and air thrust correction as a minimum and taking into account the possible correlations.

- The second matrix includes the contributions to the uncertainty of the indications. It is a diagonal matrix with the different variances of the indications on the diagonal.

- The third matrix includes the uncertainty for the model, that is, the adequacy of the approximation function to the results obtained in the calibration. It is expressed as the product of a unitary matrix and a parameter, which can be increased successively until the condition of consistency considered is met.

\subsection{Equations of air density}

With respect to the air density equation, $\rho_{\mathrm{a}}$, many equations have been eliminated since they were not useful and only the following ones have been left:

- CIPM equation 2007: reference only.

- Simplified version of the formula of the CIPM, exponential version: it has been left because, of the equations of air density that appeared in the previous version of the guide and required the measurement of environmental conditions, it is the one that has less uncertainty without be the CIPM 2007. It is given by this expression where $p$ is the atmospheric pressure in $\mathrm{Pa}, R H$ the relative humidity in $\%$ and $t$ the temperature in degrees Celsius:

$$
\rho_{\mathrm{a}}=\frac{0,34848 p-0,009 R H \exp (0,061 t)}{273,15+t}
$$

- Average air density: it was left because it is very useful if the environmental conditions are not measured and, as a novelty, its associated uncertainty, $1.2 \%$, is included.

It is given by this expression, where $\rho_{0}$ is the density of the reference air, which is $1.2 \mathrm{~kg} / \mathrm{m}^{3}, p_{0}$ is the standard atmospheric pressure, $101325 \mathrm{~Pa}, g$ the acceleration of gravity equal to $9.81 \mathrm{~m} / \mathrm{s}^{2}$ and $h$ the altitude above sea level in meters:

$$
\rho_{\mathrm{a}}=\rho_{0} \exp \left(-\frac{\rho_{0}}{p_{0}} g h_{\mathrm{SL}}\right)
$$

There is also a new way of estimating the uncertainty of the air density in a certain place by temperature variation. In order to do this, it has been considered: a maximum variation of the relative humidity of $100 \%$, the atmospheric pressure in one place has a normal distribution with standard deviation $10 \mathrm{hPa}$ and the maximum variation of the temperature in a location is included as $\Delta T$. The expression is the following: 


$$
\frac{u\left(\rho_{\mathrm{a}}\right)}{\rho_{\mathrm{a}}}=\sqrt{1,07 \times 10^{-4}+1,33 \times 10^{-6} \mathrm{~K}^{-2} \Delta T^{2}}
$$

It is very useful if you do not want to measure the environmental conditions and you have an estimate of the variation of the temperature at the site during the calibration.

\subsection{Considerations in the air buoyancy effect depending on the adjustment}

Some of the most novel aspects are the considerations in the air buoyancy effect depending on whether or not the instrument is adjusted. In general, when a load is placed on the instrument, the air buoyancy effect results in the following correction,

$$
\delta m_{\mathrm{B}}=-m_{\mathrm{cCal}}\left[\left(\rho_{\mathrm{aCal}}-\rho_{0}\right)\left(1 / \rho_{\mathrm{Cal}}-1 / \rho_{\mathrm{c}}\right)+\left(\rho_{\mathrm{aCal}}-\rho_{\mathrm{as}}\right) / \rho_{\mathrm{c}}\right]
$$

where $m_{\mathrm{cCal}}$ is the conventional mass of the load, $\rho_{\mathrm{aCal}}$ is the density of the air during the measurement, $\rho_{0}$ is the density of the reference air, which is a fixed value of $1.2 \mathrm{~kg} / \mathrm{m}^{3}$, $\rho_{\text {Cal }}$ is the density of the load, $\rho_{\mathrm{c}}$ is the reference density, which has a fixed value of $8000 \mathrm{~kg} / \mathrm{m}^{3}$, and $\rho_{\text {as }}$ is the density of the air during the adjustment of the instrument.

In principle, the last term of the equation will be annulled if the instrument is adjusted before calibration or use. As a novelty, in the new version of the guide it is established that, if the instrument is not adjusted during the calibration, this last term becomes an intrinsic part of the calibration error. This error may be important for high precision instruments, so it is also recommended that instruments with relative resolution better than $1 \times 10^{-5}$ of the full scale have to be adjusted before calibration and use.

Regarding the expression of the determination of the contribution to the uncertainty of the correction for this effect, as a novelty in the new version of the guide, a new term appears, which is derived in terms similar to the equation c.6.3-1 of the OIML R 111 [2]. It is given by the following expression, where $\rho_{\mathrm{a} 1}$ is the density of the air when the mass standards were calibrated and the suffix "Cal" is omitted,

$$
u_{\text {rel }}^{2}\left(\delta m_{\mathrm{B}}\right)=u^{2}\left(\rho_{\mathrm{a}}\right)\left(1 / \rho-1 / \rho_{\mathrm{c}}\right)^{2}+\left(\rho_{\mathrm{a}}-\rho_{0}\right)\left[\left(\rho_{\mathrm{a}}-\rho_{0}\right)-2\left(\rho_{\mathrm{a} 1}-\rho_{0}\right)\right] u^{2}(\rho) / \rho^{4}
$$

This term is very useful when both the calibration of the mass standards and the calibration of the instrument are performed at a certain altitude, since the uncertainty is reduced. Anyway, if you do not consider it the only effect that entails is an overestimation of the uncertainty.

Another novelty of the new version of the guide is the determination of the contribution to the uncertainty of this effect when the instrument is not adjusted and a maximum variation for the temperature between adjustments can be assumed. In this case it can be considered that the maximum relative uncertainty for air density depends on this variation of temperature, giving rise to the following expression,

$$
u_{\text {rel }}\left(\delta m_{\mathrm{B}}\right) \approx \sqrt{1,07 \times 10^{-4}+1,33 \times 10^{-6} \mathrm{~K}^{-2} \Delta T^{2}} \cdot \rho_{0} / \rho_{\mathrm{c}}+m p e /\left(4 m_{\mathrm{N}} \sqrt{3}\right)
$$

where mpe is the maximum permissible error of the load and $m_{\mathrm{N}}$ its nominal value.

\subsection{Uncertainty contribution due to an instrument adjustment change caused by the air density variation}


Within the uncertainty contributions in use of the instrument are those due to the influence of environmental conditions and, among these ones, we find the uncertainty contribution due to the change in the instrument's adjustment caused by the air density variation. This contribution is caused by changes in the instrument's adjustment during use due to changes in air buoyancy.

The old version of the guide said practically nothing, but, in the new version, it has been specified depending on when the instrument is adjusted.

- If it is adjusted before use, we have this expression for the relative uncertainty, which is basically derived from a second order term from the expression of the relative uncertainty of the correction by air buoyancy in relative. In this expression $\rho_{\mathrm{a}}$ is the variation of air density during use, $\rho_{\mathrm{c}}$ is the reference density, which is $8000 \mathrm{~kg} / \mathrm{m}^{3}$, and $u\left(\rho_{\mathrm{s}}\right)$ is the uncertainty of the density of the weight with which it is adjusted the instrument.

$$
u_{\text {rel }}\left(\delta R_{\text {bouy }}\right)=\frac{\Delta \rho_{\mathrm{a}}}{\rho_{\mathrm{c}}{ }^{2}} u\left(\rho_{\mathrm{s}}\right)
$$

- If the instrument is not adjusted before use, we have this other expression for the relative uncertainty where the maximum relative variation of the air buoyancy correction is considered.

$$
u_{\text {rel }}\left(\delta R_{\text {bouy }}\right)=\frac{\Delta \rho_{\mathrm{a}}}{\rho_{\mathrm{c}} \sqrt{3}}
$$

To complete this section it is sufficient to indicate which considerations can be taken into account when the variation of air density during use is not determined experimentally.

- If no information is available on what this variation is, it can be assumed that this will be a maximum of $10 \%$ of the reference air density considered as a rectangular distribution:

$$
u_{\text {rel }}\left(\delta R_{\text {bouy }}\right)=\frac{0,1 \rho_{0}}{\rho_{\mathrm{c}} \sqrt{3}}
$$

- If information is available about the maximum variation of the temperature between adjustments, the maximum relative uncertainty contribution of the air density that has been seen previously can be used:

$$
u_{\text {rel }}\left(\delta R_{\text {bouy }}\right)=\frac{\sqrt{1,07 \times 10^{-4}+1,33 \times 10^{-6} \mathrm{~K}^{-2} \Delta T^{2}} \cdot \rho_{0}}{\rho_{\mathrm{c}}}
$$

\section{Discussion}

Although there have been many changes, these changes have not been significant, in the sense that they have not changed in the essentials. Thus, the following aspects have remained invariant:

- Structure.

- Calibration method, since there are still three tests: repeatability, eccentricity and indication error.

- General expression for the determination of uncertainty.

- Determination of conventional mass and mass values.

- Typology of the instruments treated in the examples, although, as already mentioned, an additional example is included relative to the determination of the errors in front of the 
indications by means of the adjustment to a polynomial of matrix form that does not appear in the previous version.

\section{Conclusions}

This revision of the guide has clearly achieved its goal, as it has become a much clearer and more useful document, not only for calibration laboratories, but also for any user of nonautomatic weighing instruments.

\section{References}

1. EURAMET cg 18: Guidelines on the Calibration of Non-Automatic Weighing Instruments. Version 4.0

2. OIML R 111-1. Weights of classes $E_{\underline{1}}, E_{\underline{2}}, F_{\underline{1}}, F_{\underline{2}}, M_{1}, M_{1-2}, M_{\underline{2}}, M_{2-3}$ and $M_{\underline{3}}$. Part 1: Metrological and technical requirements, 2004.

3. R. Nater, A. Reichmuth, R. Schwartz, M. Borys and P. Zervos, Dictionary of Weighing Terms - A Guide to the Terminology of Weighing, Springer, Berlin, Heidelberg, 2009. ISBN 978-3-642-02013-1 